ACCEPTED MANUSCRIPT

\title{
Approximating Gecko Setae via Direct Laser Lithography
}

To cite this article before publication: Omar Tricinci et al 2017 Smart Mater. Struct. in press https://doi.org/10.1088/1361-665X/aa9e5f

\section{Manuscript version: Accepted Manuscript}

Accepted Manuscript is "the version of the article accepted for publication including all changes made as a result of the peer review process, and which may also include the addition to the article by IOP Publishing of a header, an article ID, a cover sheet and/or an 'Accepted Manuscript' watermark, but excluding any other editing, typesetting or other changes made by IOP Publishing and/or its licensors"

This Accepted Manuscript is @ 2017 IOP Publishing Ltd.

During the embargo period (the 12 month period from the publication of the Version of Record of this article), the Accepted Manuscript is fully protected by copyright and cannot be reused or reposted elsewhere.

As the Version of Record of this article is going to be / has been published on a subscription basis, this Accepted Manuscript is available for reuse under a CC BY-NC-ND 3.0 licence after the 12 month embargo period.

After the embargo period, everyone is permitted to use copy and redistribute this article for non-commercial purposes only, provided that they adhere to all the terms of the licence https://creativecommons.org/licences/by-nc-nd/3.0

Although reasonable endeavours have been taken to obtain all necessary permissions from third parties to include their copyrighted content within this article, their full citation and copyright line may not be present in this Accepted Manuscript version. Before using any content from this article, please refer to the Version of Record on IOPscience once published for full citation and copyright details, as permissions will likely be required. All third party content is fully copyright protected, unless specifically stated otherwise in the figure caption in the Version of Record.

View the article online for updates and enhancements. 


\title{
Approximating Gecko Setae via Direct Laser Lithography
}

\author{
Omar Tricinci ${ }^{*}+$, Eric V. Eason ${ }^{\ddagger}$, Carlo Filippeschi ${ }^{\dagger}$, Alessio Mondini ${ }^{\dagger}$, Barbara Mazzolai ${ }^{\dagger}$,

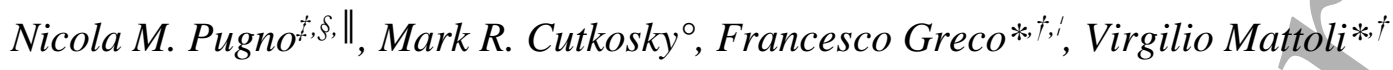

† Center for Micro-BioRobotics @ SSSA, Istituto Italiano di Tecnologia, Viale Rinaldo Piaggio 34, 56025 Pontedera, Italy.

${ }^{¥}$ Lockheed Martin Advanced Technology Center, Palo Alto, CA 94304, USA.

$\$$ Laboratory of Bio-Inspired and Graphene Nanomechanics, Department of Civil, Environmental and Mechanical Engineering, University of Trento, Via Mesiano 77, 38123 Trento, Italy.

${ }^{\S}$ School of Engineering and Materials Science, Queen Mary University of London, Mile End Road, E1 4NS London, United Kingdom.

\| Ket Lab, Edoardo Amaldi Foundation, Italian Space Agency, Via del Politecnico snc, 00133 Rome, Italy.

- Biomimetics and Dexterous Manipulation Laboratory, Center for Design Research, Department of Mechanical Engineering, Stanford University, Stanford, CA 94305 USA.

' Dept. of Life Science and Medical Bio-Science, Graduate School of Advanced Science and Engineering, Waseda University, 2-2 Wakamatsu-cho, 169-8480 Shinjuku-ku Tokyo, Japan.

KEYWORDS. Biomimetics; two-photon lithography; gecko; dry adhesion; biomimetic hierarchical materials.

\footnotetext{
ABSTRACT. The biomimetic replication of dry adhesion present in the gecko's foot has attracted great interest in recent years. All the microfabrication techniques used so far were not be able to faithfully reproduce the hierarchical and complex three-dimensional geometry of the gecko's setae, with features at the micro- and nano-scale, thus reducing the effectiveness that such type of conformal morphology could provide. By means of direct laser lithography we fabricated artificial
} 
hairs that faithfully reproduce the natural model. This technique allows the fabrication of threedimensional microstructures with outstanding results in terms of reproducibility and resolution at the micro- and nanoscale. It was possible to get very close to the morphology of the natural gecko setae, especially concerning the hierarchical shape. We designed several morphologies for the setae and studied the effects in terms of adhesion and friction performances compared to the natural counterpart, showing the interplay between morphology, dimensional scaling and materials. Direct laser lithography promises great applications in the biomimetics field, paving the way to the implementation of the concept of hierarchical bioinspired dry adhesives.

\section{Introduction}

The fabrication of smart surfaces based on biomimetic principles has attracted great interest in recent years. ${ }^{1-7}$ In particular, several studies have tried to reproduce the remarkable capability of dry adhesion that is present in the gecko's foot. ${ }^{8-22}$ Geckos can easily climb surfaces with various degrees of roughness, supporting their relatively high weight thanks to the hierarchical conformal morphology of the pads at the micro- and nano-scale. The gecko's adhesive system is directional and controllable: it provides immediate and strong adhesion when loaded in a particular direction and it requires very low force for attachment and detachment. In addition, it is "self cleaning" and does not attract dirt; hence it can be reused many times without degrading its performance. ${ }^{23}$ These characteristics would be desirable for climbing and grasping applications in robotics.

Although numerous dry adhesives have been created using lithographic and micromachining techniques, none of these fully realizes the capabilities of the gecko's adhesive system. One important reason may be that it is difficult to reproduce the complex three-dimensional morphology of the gecko's lamellae, setae and spatula with features sizes below $1 \mu \mathrm{m}$. Instead, 
lithographic processes produce arrays of pillars, wedges, stalks or "mushroom cap" features that produce useful levels of van der Waals adhesion when pressed against smooth and clean surfaces, but not on surfaces with high levels of roughness. ${ }^{24}$ For better conformability, a number of investigations have produce hierarchical features, however these usually come at the expense of decreased real area of contact, and hence reduced adhesion, on smooth surfaces. ${ }^{25}$ Here, by means of a 3D laser lithography technique, complex artificial hairs inspired by the gecko pad were fabricated, with a geometry that approximates the biological model. Direct 3D laser lithography has already demonstrated its potential in the realization of biomimetic surfaces patterned with three-dimensional features at sub-micrometric resolution. ${ }^{26-29}$ The only limiting factor of the proposed technique is related to the relative large fabrication time even if advanced 3D laser lithography systems could overcame such issue (Galvo mode, Nanoscribe GmbH). In this work we also validated the adhesive and friction performances of the artificial setae, investigating different designs and showing the crucial interplay between morphology, dimensional scaling and materials.

The study started from a detailed design of the setae, based on the anatomy of gecko pads, which has been thoroughly investigated. ${ }^{1,23}$ The gecko foot skin is composed of a multilevel structure of lamellae, setae, branches and spatulae primarily composed of $\beta$-keratin, whose elastic modulus is $1.5 \mathrm{GPa} .{ }^{30}$ The setae have a length in the range of $30-130 \mu \mathrm{m}$ and a diameter of about $5-10 \mu \mathrm{m}$, while the spatulae - the end portions which ramify from the branches - have final tips $0.5 \mu \mathrm{m}$ long, $0.2-0.3 \mu \mathrm{m}$ wide and $10 \mathrm{~nm}$ thick (Figure 1a). Each segment, thanks to its independent deformation, easily conforms and comes in close contact to solid surfaces at different levels of roughness, from the $\mathrm{mm}$ to the $\mathrm{nm}$ scale, providing a huge number of short range interactions mainly governed by van der Waals forces. ${ }^{30,31}$ In the case of Gekko gecko, each of the 
5000 setae $\mathrm{mm}^{-2}$ can ensure an average adhesion force of about $20 \mu \mathrm{N}$, although the maximum adhesion can reach a value tenfold higher, depending on the preload. ${ }^{32}$

The high aspect ratio of the stalk, the hierarchical morphology and the micro- and nanoscale features of the branches make the replication of such a complex design extremely challenging with most microfabrication techniques. The artificial setae constructed in this work are composed of I) a stalk, II) two levels of branches and III) the final spatulae (Figure 1b). Different designs were obtained by tuning the angles between the branches. We focused our investigation on the friction force and adhesive normal force of the artificial setae, which play a key role in the so-called adhesive friction or parallel adhesion that is exploited by the geckos. ${ }^{33}$

Finally, it seems worthy to remark the goal of our investigation since the fabrication of dry adhesives could also be obtained by means of a range of inorganic materials that the scientist can nowadays choose according to practical issues. Nature, on the other hand has developed a peculiar shape for the setae due to materials constraints. 

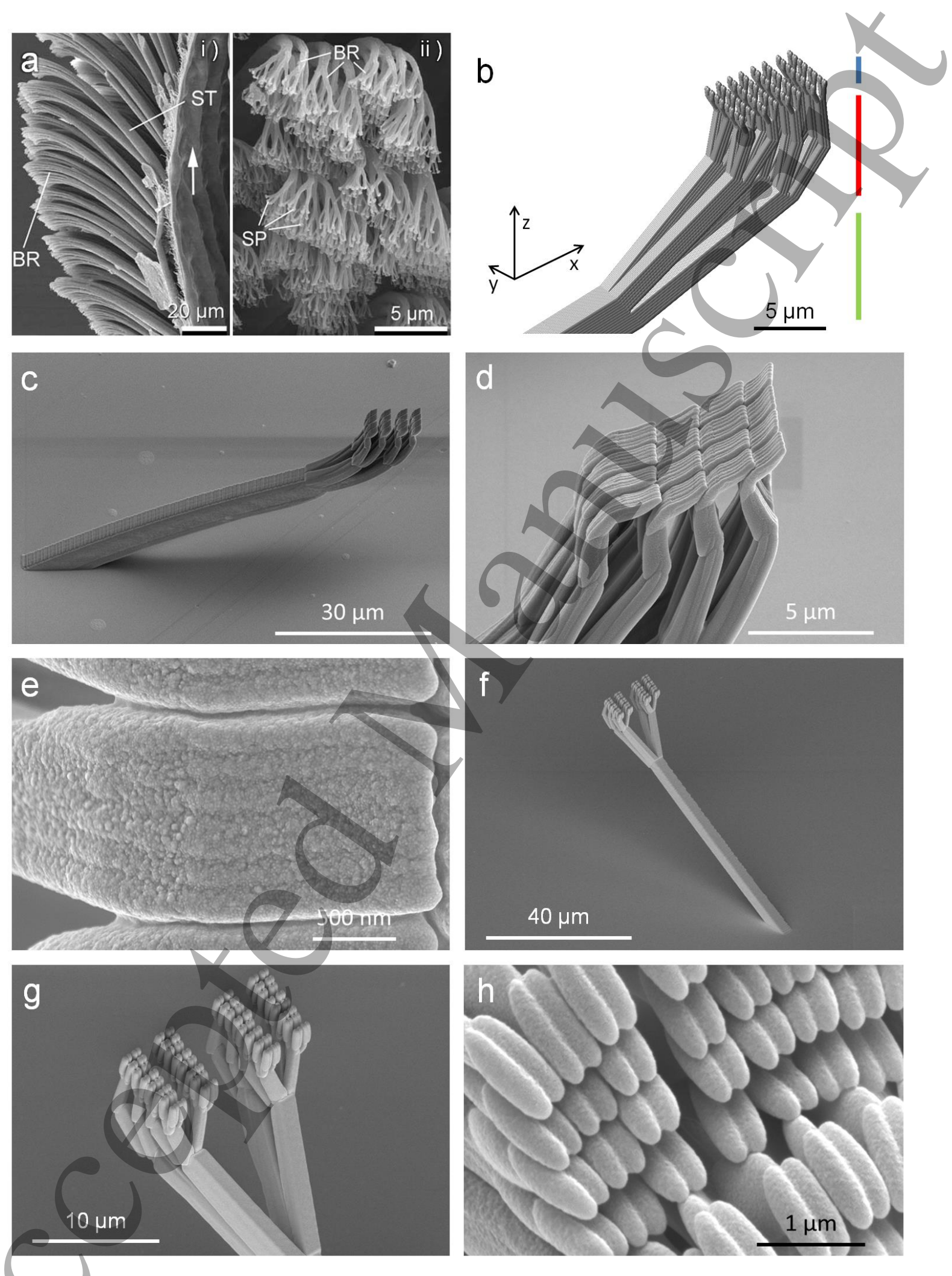

Figure 1. a) Scanning electron micrographs reporting the hierarchical adhesive structures of Gekko gecko at different magnifications; ST: seta, SP: spatula, BR: branch (adapted from H. Gao 
et al. 2005, with permission from Elsevier) ${ }^{34}$. b) Simulation of the artificial programmed setae showing the first level of branching, the second level and the final spatulae (green, red and blue bar respectively). c) First prototype of setae and details of branches (d) and spatulae ((e) adapted from O. Tricinci et al. 2017, with permission from Springer $)^{35}$. f) Example of a definitive design and details of branches in $(\mathrm{g}) . \mathrm{h}$ ) View from the top of the definitive design, showing the details of the spatulae.

\section{Experimental methods}

\subsection{Microfabrication procedure}

The setae were fabricated in IP-DiLL photoresist (Nanoscribe GmbH) by means of a Nanoscribe system (Nanoscribe $\mathrm{GmbH})$. The elastic modulus of the photoresist is 1-3 GPa, according to the laser power and the writing speed ${ }^{36}$ comparable to the natural counterpart, thus being a good candidate material for the artificial structures. A square silicon sample of side $1 \mathrm{~mm}$ was glued on a glass slide; the resist, poured on the silicon substrate, was exposed to a laser beam at a center wavelength of $780 \mathrm{~nm}$, using a writing speed of $100 \mu \mathrm{m} \mathrm{s}^{-1}$ with a power of $5.6 \mathrm{~mW}$ (Calman laser source). The sample was developed for 20 min in SU-8 Developer (MicroChem Corp) and rinsed in IPA and deionized water.

\subsection{Design of artificial setae}

The fabrication procedure required the design of two different models. The first model tried to reproduce the rough morphology of the gecko setae and it was used for the calibration of the main fabrication parameters such as the laser power and the writing speed (Figure S1 in SI). The second model was designed, in several slightly different variant morphologies, in order to reproduce the effective shape of the natural setae with a higher level of details. The structures fabricated according to this type of model were used for the adhesion and friction tests. In both cases the tips have been modeled as paths of coordinates in Matlab. The sorting strategy is based on a breadth- 
first algorithm, in which all adjacent edges at the same depth from the base are written before moving to the next level of edges that are further from the base. The programmed stalk has a nominal length of $60 \mu \mathrm{m}$ and a square section with nominal sides of $3.4 \mu \mathrm{m}$; it forms an angle of $30^{\circ}$ relative to the substrate. The branches grow of about $20 \mu \mathrm{m}$ along the direction perpendicular to the substrate. There are 16 rectangular final tips with sides of $1 \mu \mathrm{m}$ and $1.2 \mu \mathrm{m}$ (Figure 1c-e). Once the lithographic parameters have been defined, they have been used for the fabrication of the setae for the experimental tests. A Matlab program was written for the implementation of different geometrical parameters of the setae which may affect the adhesion properties. In the definitive model the stalk has a nominal length of $60 \mu \mathrm{m}$ and it has a square section with nominal sides of $2.8 \mu \mathrm{m}$ and it forms an angle of $30^{\circ}$ relative to the substrate. There are three levels of branches that end with 128 tips in total, each of $300 \mathrm{~nm}$ in thickness and $1 \mu \mathrm{m}$ in length (Figure $1 \mathrm{f}-\mathrm{h}$ ).

\subsection{Friction and adhesion tests}

The adhesion and friction tests were carried out under an optical microscope (KH-7700 digital microscope, Hirox). The microforce sensor (FemtoTools $\mathrm{GmbH}$ ) was mounted on a micromanipulator, while the silicon sample with the artificial gecko setae was fixed on the tip of a nanomanipulator (Kleindiek Nanotechnik GmbH), as shown in Figure 2a. The surface of the sensing component was silicon. The sample and the sensor were properly aligned under the microscope (Figure 2b). The cycles of the tests were controlled by means of dedicated software code written with Visual Basic (Microsoft Corp.) and data were acquired at a frequency of 10-2 Hz. Since the microforce sensor operates only along the direction of its axis, and therefore it was not possible to measure at the same time the perpendicular preload and the effective friction force, a calibration procedure for the estimation of the perpendicular preload as a function of the bending of the setae was required. 
For the evaluation of the friction on silicon, the test cycles consisted of: i) approaching and perpendicular preloading, ii) parallel displacement of about $5 \mu \mathrm{m}$, iii) sliding of the setae along the surface of the microforce sensor during which the parallel adhesive force is measured, and iv) final perpendicular detachment (Figure 2c). The measurement of the friction force was carried out in a quasi-static condition, at a speed of $35 \mathrm{~nm} \mathrm{~s}^{-1}$.

The test for the perpendicular adhesion of the artificial setae was carried out on two different target surface materials with different stiffness: silicon, that is hard, and polydimethylsiloxane (PDMS), that is softer. The PDMS surface was obtained by dip coating the silicon sensor. The samples were moved against the sensor with a speed of $5 \mu \mathrm{m} \mathrm{s}^{-1}$, until reaching a preset load that varied depending on the geometry and surface tested. Then a parallel slide of $10 \mu \mathrm{m}$ was imposed to provide the rearrangement of the tips. Finally the samples were detached (in the perpendicular direction) with a speed of $5 \mu \mathrm{m} \mathrm{s}^{-1}$. 


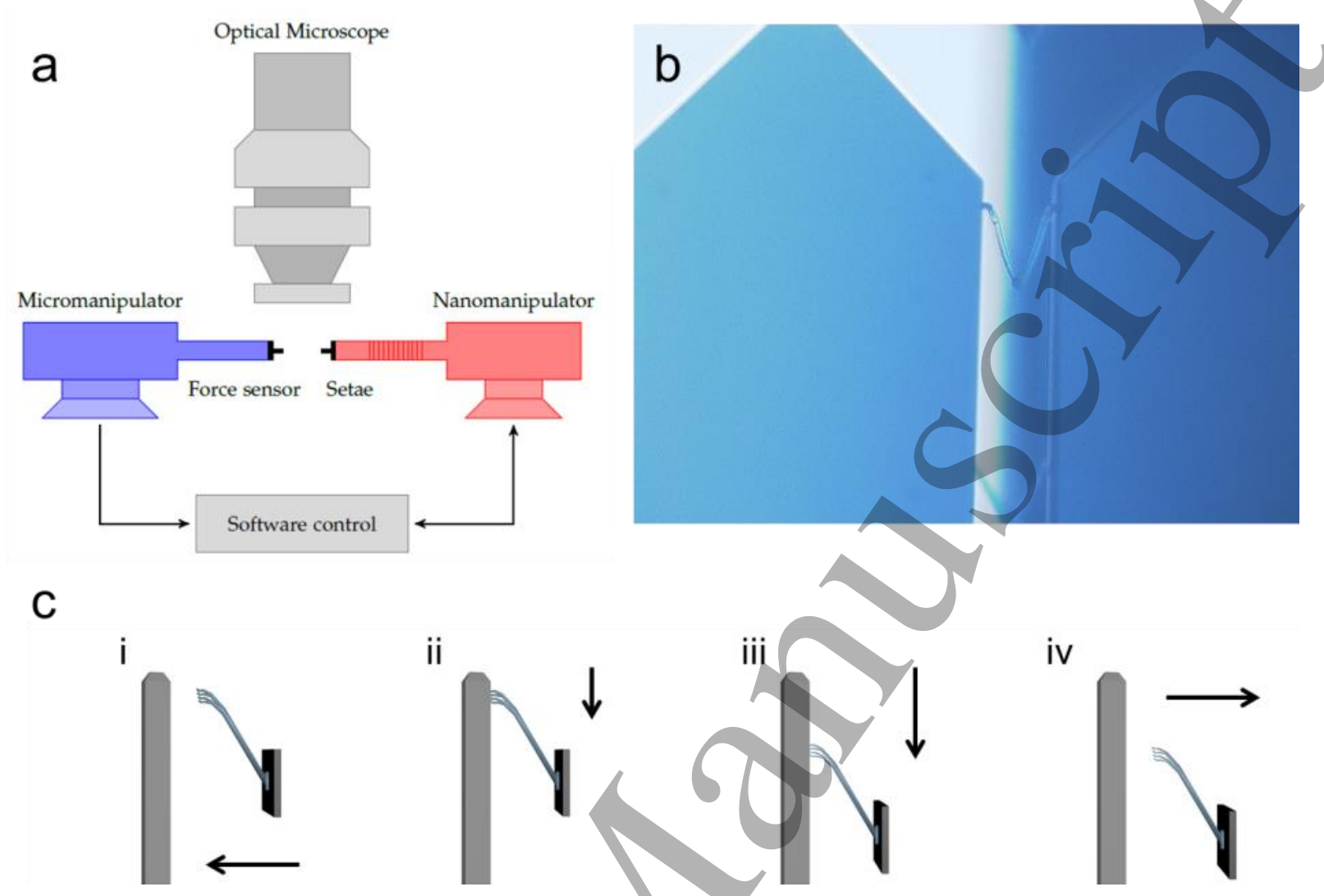

Figure 2. a) Scheme of the experimental setup for the estimation of the friction and adhesion force. b) Optical image of the artificial microfabricated setae aligned with the sensor cantilever. c) Phases of the cycle of friction tests: (i) approach and perpendicular preload; (ii) parallel preload; (iii) parallel quasi-static sliding; (iv) detachment.

\section{Results and discussion}

Ten different designs of the setae obtained by changing the angles between the branches (labelled from A to J, Figure 3a) were tested, assessing the effect of density/proximity of spatulae on the friction and normal adhesive forces. The results of the microfabrication procedure are illustrated in Figure $\mathrm{S} 2-3$ in SI.

In Figure $3 b$ the typical behaviour of the parallel frictional force as a function of time is depicted. A prominent factor affecting the friction is the preload imposed on the setae. For this reason, emulating the behaviour of the gecko during its walk, both perpendicular and parallel preload 
forces were applied. ${ }^{32}$ The perpendicular preload force was applied perpendicularly against the substrate while the parallel preload was applied by means of a small parallel displacement; this increases the number of the contact points as the spatulae lay over and conform to the substrate. Experimental data of the friction force vs perpendicular preload (Figure S4 in SI) are compatible with a linear relationship that would be expected in frictional phenomena.

Designs I, B and H ensure good friction (maximum slope of the curve in Figure S4 in SI), in absolute terms (Figure 3c). To confirm the effectiveness of the hierarchical configuration, we carried out the same experiment on a control sample consisting of a single cylindrical pillar composed by 4 segments with the same inclination and section area of the corresponding level of the tested samples and with a semi-spherical tip (Figure S5 in SI and Figure 3e). Such shape was designed in order to have the same contact area of the samples, ensuring a permanent contact that was very hard to achieve with a flat control surface. Comparing the results of the friction (at $5 \mu \mathrm{N}$ of perpendicular preload) of the control with those of the hierarchical samples, we found that the control sample was weaker, demonstrating that the hierarchical configuration was more effective; in our case, the artificial setae produced forces 6-9 times higher than the control.

To compare the performances of the artificial setae with the natural ones, it is necessary to normalize their contact area. In particular, the range of interest is around $5 \mu \mathrm{N}$ of perpendicular preload, since this is the typical value observed in gecko setae, which, in this condition, can provide an average force of $20 \mu \mathrm{N}$, that is a normalized force of $0.1 \mathrm{~N} / \mathrm{mm}^{2}{ }^{32}$ The area covered by the artificial spatulae ranges between about $35 \%$ and $80 \%$ (depending on the specific design considered) of the area of the natural ones, due to the different angles between the branches. Broader angles between the branches (as in cases $\mathrm{H}$ and I) produce a large area of contact and friction force, as shown in Figure 3c, but a lower effectiveness when normalized by area (Fig. 3d). 
2

3

4

5

6

7

8

Conversely, a configuration with closer spatulae (as in cases A and B) ensures high values of force per unit area, comparable with the natural setae since the spatulae have greater density. The best design solution should have an increase of the density of the artificial spatulae while avoiding selfsticking or bunching: this can be obtained by reducing the dimensions of the spatula, which is exactly what happens in the natural gecko setae.

Although the friction force of artificial setae is comparable to the natural counterpart, their conformability to every type of surface is intrinsically limited, since they do not include morphological features down to the tens of nanometers, which are instead present in gecko setae. ${ }^{32}$ We note also that the surface of the sensor used for the tests plays a role in the friction; although it is flat it is not smooth at the nanometer scale but is characterized by a roughness with features comparable to the natural spatulae (Figure S6 in SI). For all these reasons it is expected that artificial setae will exert lower forces than gecko setae. 

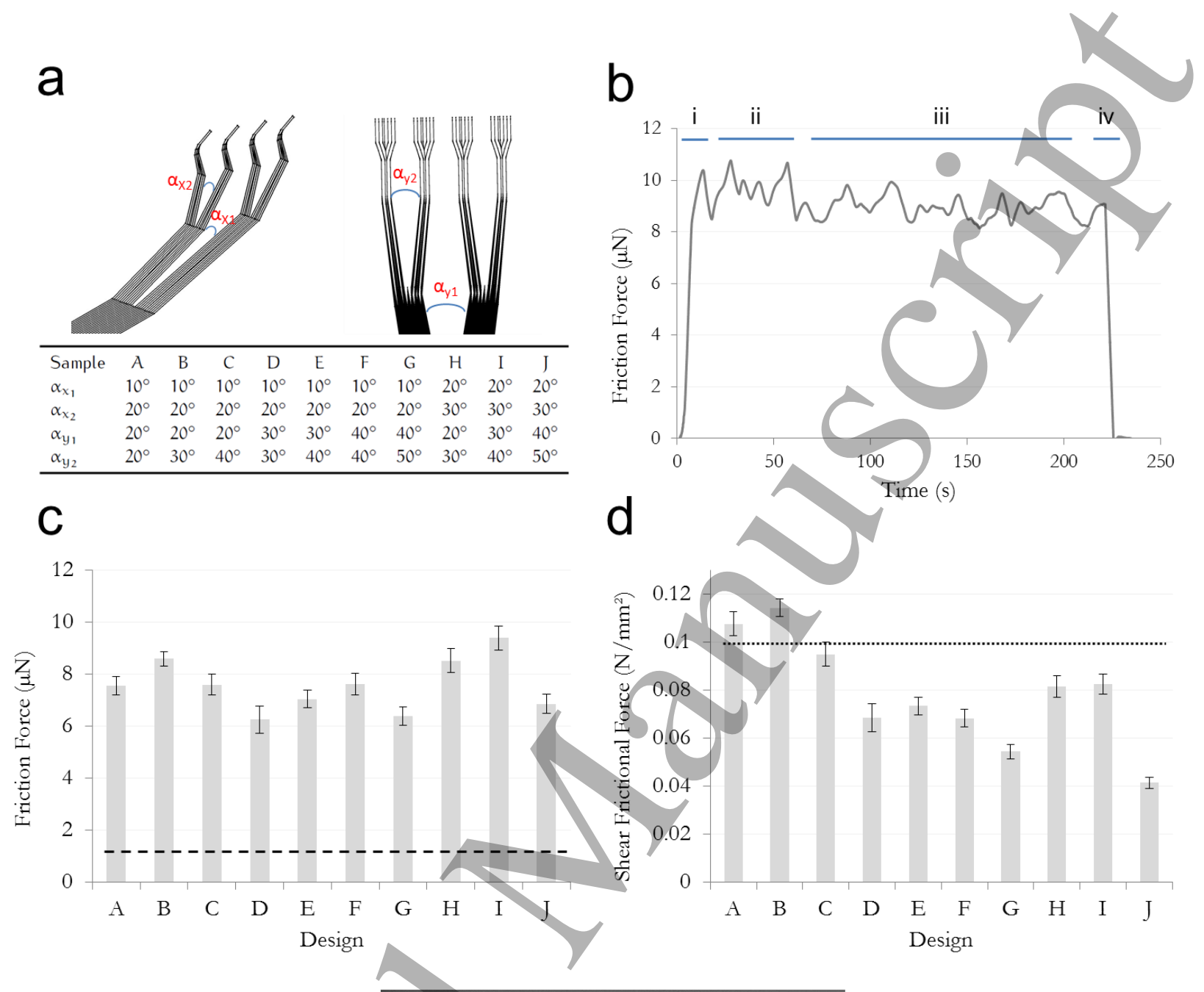
$(1.2 \mu \mathrm{N})$. d) Friction force normalized to the actual area of each artificial setae ( $5 \mu \mathrm{N}$ of perpendicular preload); the dotted line represents the performance of the natural setae $(0.1$ $\left.\mathrm{N} / \mathrm{mm}^{2}\right){ }^{32}$ e) Control sample with hemispherical tip.

In order to investigate this aspect we carried out the second experiment in/which we tested the normal adhesion of the artificial setae. In this case, it has to be considered that in the artificial setae the spatulae are a series of lines obtained by extruding a solid ellipsoid voxel, so that the terminal part of the tips involved in the contact process can be approximated to a hemisphere. For this reason the interaction of each feature in the spatulae with a flat surface can be modelled as that of a sphere of radius $R$. In this condition van der Waals interaction energy $W_{V D W}$ between a sphere and a flat surface is: ${ }^{37}$

$W_{V D W}=-\frac{A R}{6 D}$

where $D$ is the distance between the body and the surface $(D \ll R)$ and $A$ is the Hamaker constant in the range of $10^{-20}-10^{-19} \mathrm{~J}\left(A=\pi^{2} C \rho_{1} \rho_{2}\right.$, where $\rho_{1}$ and $\rho_{2}$ are the number of atoms per unit volume in the two interacting bodies). ${ }^{37}$ The related van der Waals normal force is:

$F_{V D W}=-\frac{A R}{6 D^{2}}$

The van der Waals normal force between the setae and a flat surface is calculated as:

$F_{V D W}=-\frac{A R n_{t} n_{s}}{6 D^{2}}$

where $n_{t}$ and $n_{s}$ are the number of tips in the spatulae and the number of spatulae in the setae, respectively 2 and $64 ; D$ is an atomic gap distance of $0.3 \mathrm{~nm}$. The value of $\mathrm{R}$ was chosen to be 150 $\mathrm{nm}$ which represents the maximum radius of the ellipsoid along the longitudinal direction, thus providing a superior limit of confidence. According to this model, we can estimate the normal adhesion force for this type of artificial setae in the range from 0.36 to $3.6 \mu \mathrm{N}$. 
We tested the normal adhesion of the artificial setae (designs with the closest (A) and broadest (J) branches) to two different target surface materials: silicon and polydimethylsiloxane (PDMS). PDMS was chosen for its relative softness, thus trying to find a material surface able to help the setae to better conform. In the case of design A each sample consisted of six setae, while in the case of the design $\mathrm{J}$ each sample had 4 setae. During this step, if adhesion occurred, the normal force flipped sign from positive to negative because the attached setae pulled the sensor while being retracted. Figure 4 summarizes the results obtained in normal adhesion tests. A bare Si surface was first considered as the target surface. The combination of design A against the Si surface did not produce any measurable adhesion (Figure 4a) since the closely arranged tips did not properly interact with the rigid silicon surface. A similar result was observed in the case of design $\mathrm{J}$ against $\mathrm{Si}$, even though the tips had an apparently good configuration (Figure $4 \mathrm{~b}$ ). Several peaks were observed during the force increase/decrease steps, underlining how the tips had the time to adjust their conformation over the surface of the sensor. A second set of experiments considered PDMS as the target surface, obtained by dip coating the silicon sensor. The combination of design A against PDMS produced measureable adhesion (Figure 4d) since the high compliance of the PDMS was enough to provide a proper interaction with the tips. The adhesion force recorded was $1.88 \pm 0.11 \mu \mathrm{N}$, with a mean adhesion force of $0.31 \mu \mathrm{N}$ per seta. This small value is not within the interval predicted by the mathematical model. The reason for such an apparently weaker adhesion could be that not all the tips were in contact with the surface of the sensor, or that each tip was not fully aligned with the surface. Improved adhesion was observed in the case of design J against PDMS (Figure 4e). Rearrangement of tips is again observed as a short sawtooth profile in the graphic. The recorded adhesion force was $1.82 \pm 0.15 \mu \mathrm{N}$, which means a mean single seta force of $0.46 \mu \mathrm{N}$, inside the predicted value range and considerably higher than 
design A. The result is promising since, as already evidenced for design A, not all of the setae may have made perfect simultaneous contact with the surface of the sensor. In contrast, the control sample showed no measureable adhesion on either the Si or PDMS surfaces (Figure 4c,f). Although the obtained values are far from the performance of the natural setae, we were able to measure, for the best configuration, around $25 \mathrm{~g} / \mathrm{cm}^{2}$ of adhesion that is not only due to the target material (PDMS) adhesiveness. In fact, from these tests we can deduce that broader artificial spatulae are able to exert improved adhesion forces with respect to narrower designs since they can better conform to the surface; nevertheless a higher density of spatulae per surface area would be required to obtain an effective force comparable to the gecko setae. These two requirements must be balanced according to the minimum size of features that can be fabricated with the proposed technique.

Additionally, it is important to remark that, while the present study mainly focused on structural features as a means to tune adhesion of artificial setae, the material composition (both of the artificial setae and target surface) plays a role in the adhesion. Focusing on this aspect, the effective elastic modulus represents a critical requirement: although $\beta$-keratin and IP-DiLL have comparable elastic modulus, the effective modulus is lower in the natural setae since they have a slimmer shape respect to the artificial ones. Our structures are still slightly too rigid to proper conform even on a flat surface. A soft material placed at least on the tips would be of some help.

This consideration suggests the possibility of fabricating the artificial setae with different structural materials or performing a surface functionalization to optimize their smart adhesive behaviour for specific target sürfaces. 

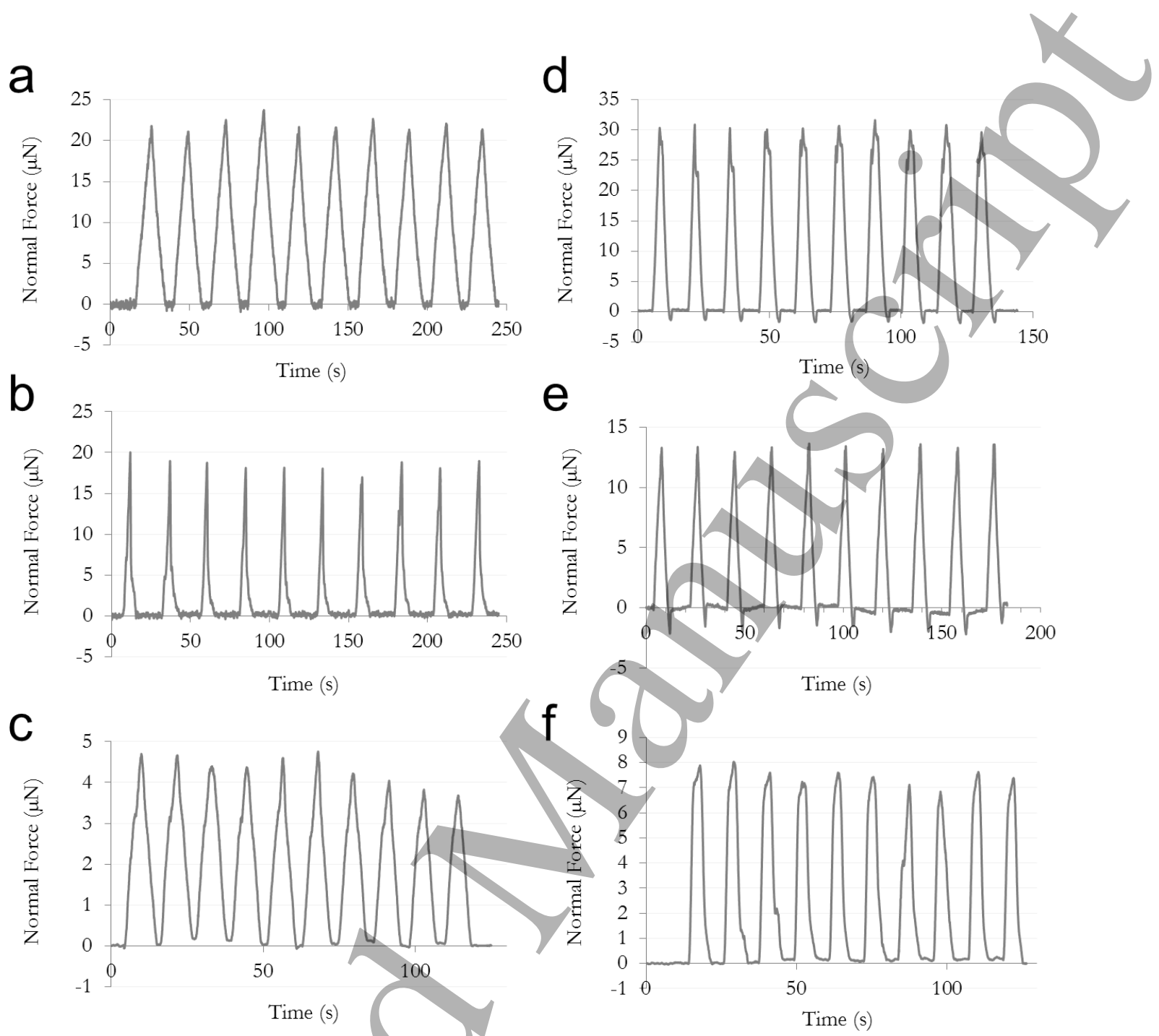

Figure 4. Normal adhesion tests of artificial setae with different designs against target surfaces: a) Normal force on Si of design A (perpendicular preload $20 \mu \mathrm{N}$ ) and in (b) design $\mathrm{J}$ (perpendicular preload $22 \mu \mathrm{N}$ ) and in (c) the control (perpendicular preload $4.5 \mu \mathrm{N}$ ); d) normal force on PDMS of design A (perpendicular preload $30 \mu \mathrm{N}$ ) and in (e) design $\mathrm{J}$ (perpendicular preload $13 \mu \mathrm{N}$ ) and in (f) the control (perpendicular preload $8 \mu \mathrm{N}$ ). 


\section{Conclusion}

In conclusion, gecko foot-like microstructures fabricated by means of direct laser lithography have been presented. These biomimetic structures reproduced with unprecedented faithfulness the structural microscale features of the animal model. The artificial setae were fabricated with different designs to investigate how their morphology influences their adhesive performance. Adhesion tests of the artificial setae developed here provided meaningful insight into their structure/property relationship. We investigated the friction force and adhesive normal force of the artificial setae, which are at the basis of the frictional adhesion friction in the geckos' toe.

In particular, friction forces similar to the natural model were obtained using optimized designs. On the other hand, adhesive force of the artificial setae is still/weaker than in the geckos' setae, due to the mechanical properties of the structures. To achieve higher forces and reach the goal of emulating the natural model, the interplay of morphology, dimensional scaling and materials involved should be considered. This work represents a first step in the direction of artificial biomimetic microstructures, with nanometric features, that closely mimic the morphology of the natural gecko setae. Selecting new structural materials would be in principle possible to enhance and optimize the adhesion on virtually every kind of target surface.

\section{ASSOCIATED CONTENT}

Supporting Information. Some more details about artificial setae fabrication and experimental procedures for adhesion tests are given. Related Figures, are also included. This material is available free of charge via the Internet at http://pubs.acs.org. 


\section{AUTHOR INFORMATION \\ Corresponding Authors \\ *E-mail: omartricinci@gmail.com \\ *E-mail: francesco.greco@iit.it \\ *E-mail: virgilio.mattoli@iit.it}

\section{Acknowledgments}

N.M.P. is supported by the European Commission H2020 under the Graphene Flagship Core 1 No. 696656 (WP14 "Polymer Composites"), and FET Proactive "Neurofibre" Grant No. 732344. F.G. acknowledges financial support from Top Global University Project (Unit for Energy and Nanomaterials) at Waseda University from MEXT Japan.

\section{References}

(1) Bhushan B 2009 Biomimetics: Lessons from Nature-an Overview. Philos. Trans. R. Soc. Math. Phys. Eng. Sci. 367 (1893) 1445-1486

(2) Koch K, Bhushan B and Barthlott W 2009 Multifunctional Surface Structures of Plants: An Inspiration for Biomimetics. Prog. Mater. Sci. 54 (2) 137-178

(3) Kinoshita S, Yoshioka S and Kawagoe K 2002 Mechanisms of Structural Colour in the Morpho Butterfly: Cooperation of Regularity and Irregularity in an Iridescent Scale. Proc. R. Soc. Lond. B Biol. Sci. 269 (1499) 1417-1421

(4) Dean B and Bhushan B 2010 Shark-Skin Surfaces for Fluid-Drag Reduction in Turbulent Flow: A Review. Philos. Trans. R. Soc. Math. Phys. Eng. Sci. 368 (1929) 4775-4806

(5) Stavenga D G, Foletti S, Palasantzas G and Arikawa K 2006 Light on the Moth-Eye Corneal Nipple Array of Butterflies. Proc. R. Soc. B Biol. Sci. 273 (1587) 661-667 
(6) Jagota A and Hui C Y 2011 Adhesion, Friction, and Compliance of Bio-Mimetic and BioInspired Structured Interfaces. Mater. Sci. Eng. R Rep. 72 (12) 253-292

(7) Hou Y, Yu M, Chen X, Wang Z, and Yao S 2015 Recurrent Filmwise and Dropwise Condensation on a Beetle Mimetic Surface. ACS Nano 9 (1) 71-81

(8) Kamperman M, Kroner E, del Campo A, McMeeking R M and Arzt E 2010 Functional Adhesive Surfaces with "Gecko" Effect: The Concept of Contact Splitting. Adv. Eng. Mater. 12 (5) 335-348

(9) Autumn K, Niewiarowski P H and Puthoff J B 2014 Gecko Adhesion as a Model System for Integrative Biology, Interdisciplinary Science, and Bioinspired Engineering. Annu. Rev. Ecol. Evol. Syst. 45 (1) 445-470

(10) Geim A K, Dubonos S V, Grigorieva I V, Novoselov K S and Zhukov A A Shapoval, S. Y. 2003 Microfabricated Adhesive Mimicking Gecko Foot-Hair. Nat. Mater. 2 (7) 461-463

(11) Northen M T and Turner K L 2005 A Batch Fabricated Biomimetic Dry Adhesive. Nanotechnology 16 (8) 1159-1166

(12) Lee H, Lee B P and Messersmith P B 2007 A Reversible Wet/dry Adhesive Inspired by Mussels and Geckos. Nature 448 (7151) 338-341

(13) Lu G, Hong W, Tong L, Bai H, Wei Y and Shi G 2008 Drying Enhanced Adhesion of Polythiophene Nanotubule Arrays on Smooth Surfaces. ACS Nano 2 (11) 2342-2348

(14) Murphy M P, Kim S and Sitti M 2009 Enhanced Adhesion by Gecko-Inspired Hierarchical Fibrillar Adhesives. ACS Appl. Mater. Interfaces 1 (4) 849-855

(15) Lee J, Bush B, Maboudian R and Fearing R S 2009 Gecko-Inspired Combined Lamellar and Nanofibrillar Array for Adhesion on Nonplanar Surface. Langmuir 25 (21) 12449-12453

(16) Greiner C, Arzt E and del Campo A 2009 Hierarchical Gecko-Like Adhesives. Adv. Mater. 21 (4) 479-482

(17) Kim T, Jeong H E, Suh K Y and Lee H H 2009 Stooped Nanohairs: GeometryControllable, Unidirectional, Reversible, and Robust Gecko-like Dry Adhesive. Adv. Mater. 21 (22) 2276-2281

(18) Kim T, Pang C and Suh K Y 2009 Shape-Tunable Polymer Nanofibrillar Structures by Oblique Electron Beam Irradiation. Langmuir 25 (16) 8879-8882

(19) Mengüç Y, Yang S Y, Kim S, Rogers J A and Sitti M 2012 Gecko-Inspired Controllable Adhesive Structures Applied to Micromanipulation. Adv. Funct. Mater. 22 (6) 1246-1254

(20) Jeong H E, Kwak M K and Suh K Y 2010 Stretchable, Adhesion-Tunable Dry Adhesive by Surface Wrinkling. Langmuir 26 (4) 2223-2226

(21) Hawkes E W, Eason E V, Asbeck A T and Cutkosky M R 2013 The Gecko's Toe: Scaling Directional Adhesives for Climbing Applications. IEEEASME Trans. Mechatron. 18 (2) 518-526 
(22) King D R, Bartlett M D, Gilman C A, Irschick D J and Crosby A J 2014 Creating GeckoLike Adhesives for "Real World" Surfaces. Adv. Mater. 26 (25) 4345-4351

(23) Gecko adhesion: evolutionary nanotechnology | Philosophical Transactions of the Royal Society of London A: Mathematical, Physical and Engineering Sciences http://rsta.royalsocietypublishing.org/content/366/1870/1575.short (accessed May 5, 2017)

(24) Human climbing with efficiently scaled gecko-inspired dry adhesives $\mid$ Journal of The Royal Society Interface http://rsif.royalsocietypublishing.org/content/12/102/20140675.short (accessed Apr 7, 2017)

(25) Kwak M K, Pang C, Jeong H-E, Kim H-N, Yoon H, Jung H-S and Suh K-Y 2011 Towards the Next Level of Bioinspired Dry Adhesives: New Designs and Applications. Adv. Funct. Mater. 21 (19) 3606-3616

(26) Marino A, Filippeschi C, Mattoli V, Mazzolai B and Ciofani G 2015 Biomimicry at the Nanoscale: Current Research and Perspectives of Two-Photon Polymerization. Nanoscale 7 (7) 2841-2850

(27) Tricinci O, Terencio T, Mazzolai B, Pugno N M, Greco F and Mattoli V 2015 3D Micropatterned Surface Inspired by Salvinia Molesta via Direct Laser Lithography. ACS Appl. Mater. Interfaces 7 (46) 25560-25567

(28) Bernardeschi I, Tricinci O, Mattoli V, Filippeschi C, Mazzolai B and Beccai L 2016 ThreeDimensional Soft Material Micropatterning via Direct Laser Lithography of Flexible Molds. ACS Appl. Mater. Interfaces 8 (38) 25019-25023

(29) Röhrig M, Thiel M, Worgull M and Hölscher H 2012 3D Direct Laser Writing of Nanoand Microstructured Hierarchical Gecko-Mimicking Surfaces. Small 8 (19) 3009-3015

(30) Autumn K, Majidi C, Groff R E, Dittmore A and Fearing R 2006 Effective Elastic Modulus of Isolated Gecko Setal Arrays. J. Exp. Biol. 209 (18) 3558-3568

(31) Autumn K, Sitti M, Liang Y A, Peattie A M, Hansen W R, Sponberg S, Kenny T W, Fearing R and Israelachvili J N Full, R. J. 2002 Evidence for van Der Waals Adhesion in Gecko Setae. Proc. Natl. Acad. Sci. 99 (19) 12252-12256

(32) Autumn K, Liang Y A, Hsieh S T, Zesch W, Chan W P, Kenny T W, Fearing R and Full R J 2000 Adhesive Force of a Single Gecko Foot-Hair. Nature 405 (6787) 681-685

(33) Autumn K, Dittmore A, Santos D, Spenko M and Cutkosky M 2006 Frictional Adhesion: A New Angle on Gecko Attachment. J. Exp. Biol. 209 (18) 3569-3579

(34) Gao H, Wang X, Yao H, Gorb S and Arzt E 2005 Mechanics of Hierarchical Adhesion Structures of Geckos. Mech. Mater. 37 (2) 275-285

(35) Tricinci O, Eason E V, Filippeschi C, Mondini A, Mazzolai B, Pugno N M, Cutkosky M R, Greco F and Mattoli V 2017 Dry Adhesion of Artificial Gecko Setae Fabricated via Direct Laser Lithography. In Biomimetic and Biohybrid Systems; Lecture Notes in Computer Science; Springer, Cham 631-636 
(36) Lemma E D, Rizzi F, Dattoma T, Spagnolo B, Sileo L, Qualtieri A, Vittorio M D and Pisanello F 2017 Mechanical Properties Tunability of Three-Dimensional Polymeric Structures in Two-Photon Lithography. IEEE Trans. Nanotechnol. 16 (1) 23-31

(37) Israelachvili J N 2011 Intermolecular and Surface Forces: Revised Third/Edition; Academic Press

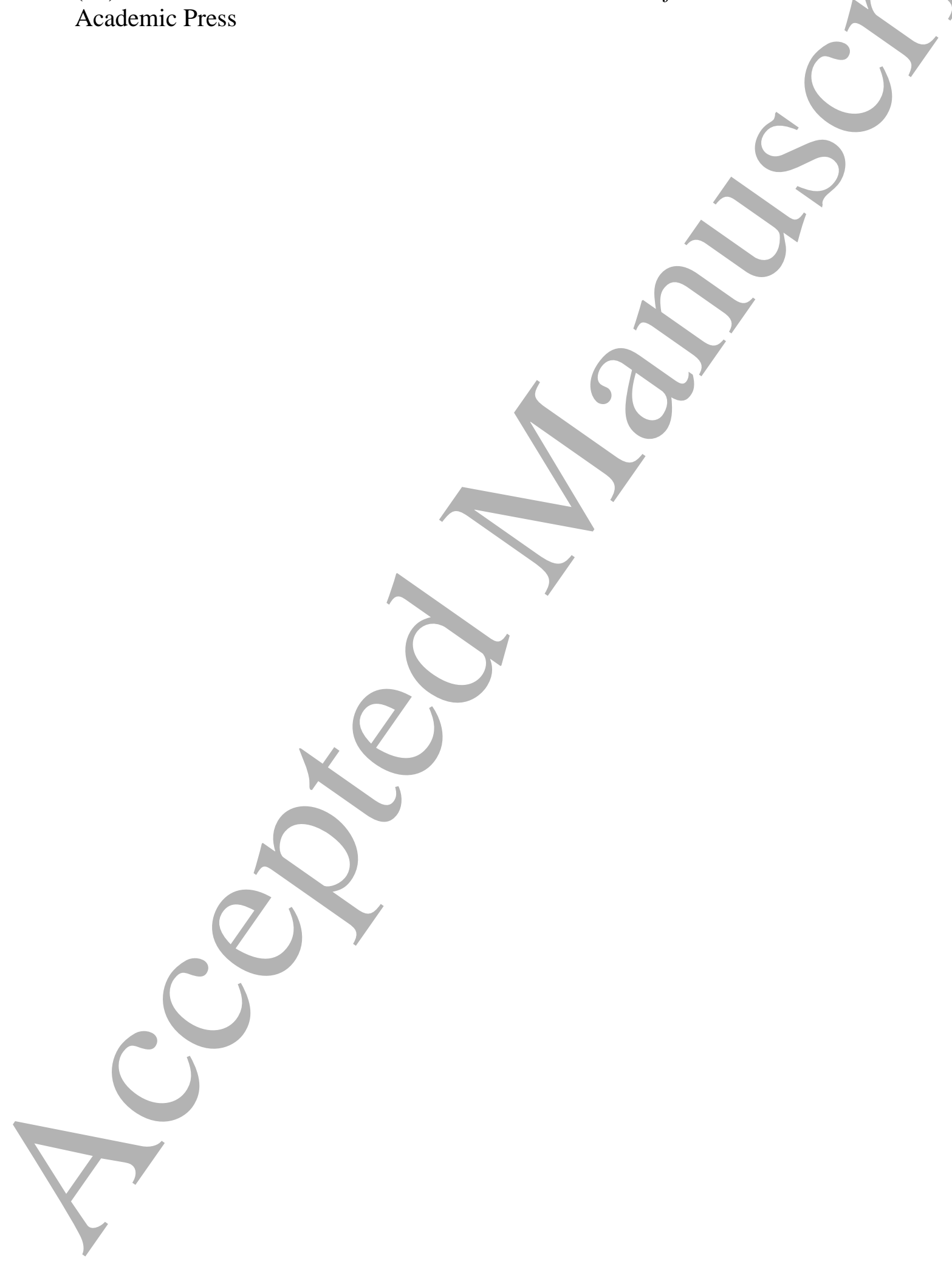

